\title{
Influence of antibiotic pressure on multi- drug resistant Klebsiella pneumoniae colonisation in critically ill patients
}

\author{
Jesus Ruiz', Monica Gordon', Esther Villarreal', Juan Frasquet², María Ángeles Sánchez³, María Martín',
} Álvaro Castellanos ${ }^{3}$ and Paula Ramirez ${ }^{3^{*}}$

\begin{abstract}
Background: The aim of this study is to evaluate the risk factors for colonisation by multidrug resistant (MDR) $K$. pneumoniae in a critical care unit and the relationship between colonisation and the antibiotic pressure exerted by the antimicrobial treatments received by patients.

Methods: A prospective observational was designed. Patients admitted for more than $48 \mathrm{~h}$ to an intensive care unit were included. Samples for surveillance cultures were obtained from all the patients upon admission and once a week. The association between risk factors and colonisation by MDR K. pneumoniae was determined by logistic regression. A Cox regression model was used to evaluate the effect of the use of antimicrobials on the colonisation rate. An ARMIA model was used to investigate the association between the incidence of colonisation by MDR strains and the global consumption of antimicrobials in the unit.

Results: One thousand seven hundred twenty-five patients were included, from which 308 (17.9\%) were positive for MDR K. pneumoniae. In the multivariate analysis, hospitalisation for longer than 7 days together with respiratory infection and administration of any antibiotic was associated with increased MR K. pneumoniae colonisation. Patients who received antibiotics for more than $48 \mathrm{~h}$ were colonised earlier than patients who did not receive antibiotic treatment [HR: 2.16 (95\%Cl:1.55-3.03)]. The ARIMA model found a significant association between the monthly colonisation rate for MR K. pneumoniae and the consumption of cephalosporins and carbapenems in the previous month.
\end{abstract}

Conclusion: Individual antibiotic administration and the global antibiotic pressure of cephalosporins and carbapenems are associated to an increased colonisation by MDR K. pneumoniae strains.

Keywords: K pneumoniae, Antibiotic, Critcal care, Multidrug resistance, Colonization

\section{Background}

Klebsiella pneumoniae is now one of the microorganisms most commonly associated with healthcare and nosocomial care-associated infections, mainly in patients admitted to critical care units [1-3]. From the first time Klebsiella spp. isolates were described as producing extended-spectrum b-lactamases (ESBL) in 1983, a progressive increase in multiresistant (MR) strains of this

\footnotetext{
* Correspondence: ramirez_pau@gva.es

${ }^{3}$ Intensive Care Unit, Hospital Universitario y Politécnico La Fe, Valencia, Spain Full list of author information is available at the end of the article
}

species has been observed worldwide [4]. The increase in the number of hospital infections caused by MR strains of $K$. pneumoniae is especially worrisome, as more than $30 \%$ of episodes of nosocomial bacteraemia caused by this species are now MR strains, reaching around $45 \%$ of episodes in critically ill patient [5]. The demonstrated association between resistance and morbimortality, hospital stay length, and health costs [6,7], as well as the limited therapeutic options available, highlights the importance of controlling and minimising the expansion of this species.

The description of the risk factors associated with infection by MR strains of species such as Pseudomonas

(c) The Author(s). 2019 Open Access This article is distributed under the terms of the Creative Commons Attribution 4.0 International License (http://creativecommons.org/licenses/by/4.0/), which permits unrestricted use, distribution, and reproduction in any medium, provided you give appropriate credit to the original author(s) and the source, provide a link to the Creative Commons license, and indicate if changes were made. The Creative Commons Public Domain Dedication waiver (http://creativecommons.org/publicdomain/zero/1.0/) applies to the data made available in this article, unless otherwise stated. 
aeruginosa or Acinetobacter baumannii in critical patients has been the subject of multiple studies, with previous exposure to various different antibiotics being one of its highest risk factors [8-11]. However, there is little data available about the effect of antimicrobial consumption on colonisation by MR K. pneumoniae, even though this phenomenon is of special interest because, in most cases, these have been used prior to infection $[12,13]$. Thus, the objective of this work was to evaluate the risk factors for colonisation by MR strains of $K$. pneumoniae in a critical care unit and to analyse the relationship between this colonisation and the antibiotic pressure exerted by the antimicrobial treatments received by patients, as well as by the overall consumption of antibiotics in the unit.

\section{Methods}

We designed a prospective and observational study which included adult patients admitted for more than $48 \mathrm{~h}$ to an intensive care unit (ICU) in a tertiary hospital between January 2014 and December 2016. The ICU where the study was conducted attends patients with medical pathologies; it has 24 beds and in recent years has had an average of 1592 admissions per year. Despite the introduction of various measures, since 2013 the unit has presented an endemic situation of $K$. pneumoniae colonisation, with a colonisation pressure (ratio of patients colonised with MR K. pneumoniae/total number of patients per day) of $14.8 \%$ this year. Patients admitted for less than $48 \mathrm{~h}$ and with colonisation known prior to admission to the unit, or who were colonised by MR species other than $K$. pneumoniae during their hospitalisation, were excluded from the study.

Following the internal operating protocols of our ICU, samples from all the patients in the unit were submitted for surveillance cultures upon admission and once a week thereon, to screen for MR bacteria colonisation. The samples consisted of a rectal swab, an oropharyngeal swab, and a bronchial aspirate from intubated patients. In addition, where a case of clinical infection was suspected, samples were obtained for microbiological study.

The clinical samples were processed according to our usual methodology. The cultures were incubated at $35^{\circ} \mathrm{C}$ for $48 \mathrm{~h}$, followed by a subculture on selective chromogenic medium for ESBL-producing enterobacteria and carbapenemases. The antibiogram of the colonies obtained from these cultures was identified and studied according to standardised methods used in the microbiology laboratory. Strains of $K$. pneumoniae were classified as MR following the criteria established by the European Centre for Disease Prevention and Control (ECDC) [14], considering MR strains to be those which were resistant to three or more groups of antimicrobials and carbapenemase-producing strains those isolates presenting either minimum inhibitory concentration (MIC) of $0.125 \mathrm{mg} /$ liter to meropenem and/or ertapenem and/or $1 \mathrm{mg} /$ liter to imipenem [15].

Data about the antibiotic exposure were collected during the follow-up period, both individually from the patients included in the study cohort, as well as globally in the unit. The overall consumption of antibiotics in the unit was calculated based on the criteria established by the WHO, by calculating the daily defined dose (DDD) per 100 patients per day [16]. For each of the patients included in the study, we recorded the antibacterial treatment received during their stay in the ICU, including any digestive-tract decontaminating solutions administered, until colonisation by $K$. pneumoniae was detected or the patient was discharged from the unit.

\section{Statistical analysis}

Statistical analysis was performed with the Stata (v.12.0) program. For the comparison of variables between patients with and without colonisation, chi-square or Fisher's exact tests were used for categorical variables and Student t-tests were implemented for continuous variables. The association between risk factors and colonisation by strains of MR $K$. pneumoniae was determined by logistic regression; any variables with a probability $(\mathrm{p})$ value of less than 0.2 in the univariate model were included in the multivariate model. A Cox regression model was used to evaluate the effect of the use of antimicrobials for more than $48 \mathrm{~h}$ on the colonisation rate. Furthermore, we used an autoregressive integrated moving average (ARIMA) model with monthly time periods to investigate the association between the incidence of colonisation by MR strains and the global consumption (DDD/100 stays) of antimicrobials in the unit during the same month and the previous month. Any tests with a $p<0.05$ value were considered significant.

\section{Results}

During the study period there were a total of 1994 ICU admissions whose stay exceeded $48 \mathrm{~h}$ and from whom an epidemiological surveillance sample was collected. Of these, 269 (13.5\%) were excluded because they were colonised by other MR species during their stay in the unit. These species were: Enterobacter cloacae in 108 (5.4\%) patients, $P$. aeruginosa in 68 (3.4\%), Methicillin-resistant $S$. aureus (MRSA) in 48 (2.4\%), A. baumannii in 32 (1.6\%), and other species in $13(0.7 \%)$ patients. A total of 1725 patients were included, from which a total 9042 surveillance samples were obtained; 1256 samples (13.9\%), corresponding to 308 (17.9\%) patients, were positive for MR K. pneumoniae, and of these, 536 (from 119 patients) were ESBL-producing strains, 745 (183 patients) were carbapenemase-producing strains, and 25 samples (6 patients) contained both strains. Of the total number of 
positive samples, $826(65.8 \%)$ were from rectal swabs, 291 (23.2\%) from pharyngeal swabs, and 139 (11.0\%) from bronchial aspirates.

The demographic and clinical characteristics of these patients are shown in Table 1 . The percentage of patients colonised, according to the number of weeks they stayed in the unit, as well as the percentage of new colonisations found for each week of stay, are shown in Fig. 1; most cases were identified after the second week of hospitalisation in the unit. The colonised patients had a longer mean stay length [19.3 \pm 16.1 days vs. $7.8 \pm 7.3$ days); $p<0.001]$ and a higher mortality rate $(30.8 \%$ vs. $17.1 \%$; $p<0.001)$ in the unit compared to the non-colonised patients (Table 2).

The univariate analysis showed that a stay longer than 7 days, the origin of the patient, an APACHE-II score exceeding 20, respiratory infection as the cause of admission, use of parenteral nutrition, and renal replacement techniques implemented along with the administration of any antibiotic for more than $48 \mathrm{~h}$-especially linezolid, penicillins/ b-lactamase inhibitors (BLIs), cephalosporins, carbapenems, or aminoglycosides - were associated with colonisation by strains of MR K. pneumoniae (Table 2). In the multivariate analysis (Table 3), hospitalisation for longer than 7 days together with respiratory infection, and administration of any antibiotic - particularly penicillins/BLIs and aminoglycosides- was associated with increased MR K. pneumoniae colonisation rates.

The effect of antimicrobial consumption on the speed with which patients were colonised in the unit is shown in Fig. 2. Patients who received antibiotics for more than $48 \mathrm{~h}$ were colonised earlier than patients who did not receive this treatment [hazard ratio: 2.16 (95\% CI: $1.55-$ 3.03)].

\section{Association between antimicrobial consumption and the colonisation rate in the unit}

The ARIMA model found a significant association between the monthly colonisation rate for MR K. pneumoniae and the consumption of cephalosporins and carbapenems in the previous month (Table 4). No

Table 1 Characteristics of patients included in the study

\begin{tabular}{|c|c|c|c|c|}
\hline & Total & $\begin{array}{l}\text { Patients colonized by } \\
\text { K. p. Pneumoniae }\end{array}$ & $\begin{array}{l}\text { Patients not colonized by } \\
\text { K. pPneumoniae }\end{array}$ & $p$ \\
\hline & $n=1725$ & $n=308$ & $n=1417$ & \\
\hline Age (SD) & $60.9(15.2)$ & $58.1(14.9)$ & $61.5(15.2)$ & $<0.001$ \\
\hline Male (\%) & $1097(63.6)$ & $203(65.9)$ & $895(63.2)$ & 0.364 \\
\hline Length of stay (SD) & $9.85(10.46)$ & $19.3(16.1)$ & $7.8(7.3)$ & $<0.001$ \\
\hline${ }^{1}$ ICU mMortality (\%) & $338(19.6)$ & $95(30.8)$ & $243(17.1)$ & $<0.001$ \\
\hline APACHE II (median, IQR) & $19(14-24)$ & $21(18-25)$ & $18(13-24)$ & $<0.001$ \\
\hline Hematologic disease & $120(6.9)$ & $19(6.2)$ & $101(7.1)$ & 0.531 \\
\hline Solid organ tTransplantation & $73(4.2)$ & $18(5.8)$ & $55(3.9)$ & 0.133 \\
\hline Immunosuppression & $267(15.5)$ & $60(19.4)$ & $207(14.6)$ & 0.035 \\
\hline Diabetes & $351(12.4)$ & $65(21.1)$ & $268(20.2)$ & 0.722 \\
\hline Cirrhosis & $64(3.7)$ & $18(5.8)$ & $46(3.2)$ & 0.028 \\
\hline${ }^{2} \mathrm{EPOCCOPD}$ & $351(20.3)$ & $93(30.2)$ & $258(18.3)$ & $<0.001$ \\
\hline Mechanical vVentilation (\%) & $408(23.7)$ & $85(27.6)$ & $324(22.8)$ & 0.077 \\
\hline Renal rReplacement therapy (\%) & $142(8.2)$ & $21(6.8)$ & $121(8.5)$ & 0.319 \\
\hline Parenteral nNutrition & $753(34.1)$ & $103(33.4)$ & $408(28.8)$ & 0.109 \\
\hline Origin & & & & 0.179 \\
\hline Emergency dDepartment & $793(46.0)$ & $126(40.9)$ & $667(47.1)$ & \\
\hline Hospitalization & $378(21.9)$ & $92(29.9)$ & $461(32.5)$ & \\
\hline Other hHospital & $534(30.9)$ & $90(29.2)$ & $282(19.9)$ & \\
\hline Cause of admission & & & & $<0.001$ \\
\hline Respiratory infection & $321(18.6)$ & $77(25.0)$ & $243(17.1)$ & \\
\hline Sepsis/Septic sShock & $181(10.5)$ & $24(7.8)$ & $156(11.0)$ & \\
\hline Cardiovascular disease & $567(32.9)$ & $60(19.5)$ & $507(35.8)$ & \\
\hline Other & $976(56.7)$ & $147(47.7)$ & $511(36.1)$ & \\
\hline
\end{tabular}

${ }^{1} \mathrm{ICU}:$ Intensive care unit; ${ }^{2}$ COPD: Chronic obstructive pulmonary disease 


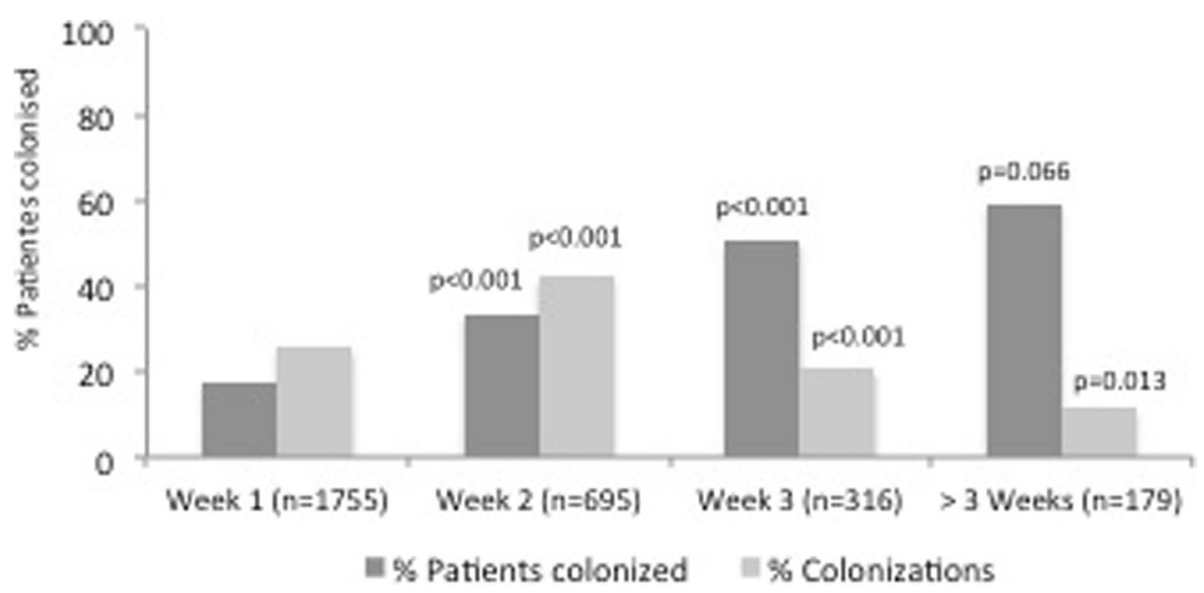

Fig. 1 Percentage of patients colonised in the unit and new colonisations according to the number of weeks of stay in the unit. P-values represent the differences in percentages of patients colonised and new colonisations between each week and the previous one

Table 2 Results from the univariante analysis

\begin{tabular}{|c|c|c|}
\hline & OR (IC95\%) & $P$ \\
\hline Age $>60$ years & $0.86(0.40-1.83)$ & 0.700 \\
\hline Length of stay $>7$ days & $6.52(4.84-8.52)$ & $<0.001$ \\
\hline APACHE $\|>20$ & $1.98(0.89-2.62)$ & 0.092 \\
\hline Hematologic disease & $0.84(0.17-3.97)$ & 0.825 \\
\hline Solid Organ Transplantation & $1.59(0.31-8.26)$ & 0.580 \\
\hline Origin & $1.38(1.21-1.57)$ & 0.168 \\
\hline Immunosuppression & $1.27(0.47-3.40)$ & 0.641 \\
\hline Diabetes & $0.63(0.26-1.56)$ & 0.320 \\
\hline Cirrhosis & $1.92(0.36-10.37)$ & 0.477 \\
\hline${ }^{1} \mathrm{COPD}$ & $0.89(0.82-1.74)$ & 0.380 \\
\hline Mechanical ventilation & $1.34(0.61-2.95)$ & 0.465 \\
\hline Renal replacement therapy & $1.66(0.91-2.14)$ & 0.134 \\
\hline Parenteral Nutrition & $2.18(1.01-4.69)$ & 0.045 \\
\hline Respiratory infection & $2.05(0.93-4.53)$ & 0.074 \\
\hline Sepsis/Septic Shock & $0.39(0.04-2.69)$ & 0.306 \\
\hline Antibiotics $>48 \mathrm{~h}$ & $5.60(3.92-7.98)$ & $<0.001$ \\
\hline Carbapenem & $1.33(0.93-1.89)$ & 0.108 \\
\hline Quinolones & $1.21(0.85-1.70)$ & 0.297 \\
\hline${ }^{2} \mathrm{IBL}$ & $3.75(2.83-4.97)$ & $<0.001$ \\
\hline Cephalosporin & $1.87(1.38-2.53)$ & $<0.001$ \\
\hline Amynoglycosides & $3.78(2.67-5.37)$ & $<0.001$ \\
\hline Linezolid & $3.02(1.21-4.17)$ & $<0.001$ \\
\hline${ }^{3} \mathrm{SDD}$ & $0.45(0.05-3.64)$ & 0.454 \\
\hline
\end{tabular}

${ }^{1}$ COPD: Chronic obstructive pulmonary disease; ${ }^{2} \mathrm{BLI}$ : b-lactamase inhibitors; ${ }^{3} \mathrm{SDD}$ : Selective digestive decontamination association between the overall consumption of antibiotics in the unit and the colonisation rate was found.

\section{Discussion}

The results of our work show that, in an endemic situation, the administration of antimicrobials, as well as the antibiotic pressure of cephalosporins and carbapenems, is related to increased colonisation by MR K. pneumoniae strains.

The expansion of MR bacteria is now one of the main concerns for critical patient units. Different authors have analysed the effect of antimicrobial consumption on colonisation by MR strains in these units, and have found a clear association between the individual consumption of antipseudomonal drugs and colonisation or infection by MR $P$. aeruginosa $[17,18]$ or $A$. baumannii strains $[9,19]$. However, even though this species is expanding, the effect of administering antimicrobials on the

Table 3 Results from the multivariate analysis

\begin{tabular}{lll}
\hline & OR (IC95\%) & $P$ \\
\hline Length of stay $>$ 7 days & $3.76(1.89-5.00)$ & $<0.001$ \\
APACHE II > 20 & $1.14(0.84-1.56)$ & 0.400 \\
Respiratory infection & $1.75(1.27-2.42)$ & 0.002 \\
Origin & $1.23(0.89-1.63)$ & 0.327 \\
Renal replacement therapy & $1.23(0.80-1.90)$ & 0.136 \\
Parenteral Nutrition & $1.32(0.96-1.80)$ & 0.087 \\
Antibiotics $>$ 48 h & $2.86(1.83-4.48)$ & $<0.001$ \\
${ }^{1}$ IBL ${ }^{1}$ BLI & $1.89(1.68-8.33)$ & $<0.001$ \\
Cephalosporin & $1.85(1.33-2.69)$ & 0.214 \\
Aminoglycosides Aminoglucósidos & $2.04(1.35-2.10)$ & 0.001 \\
Linezolid & $1.38(0.96-1.98)$ & 0.098 \\
Carbapenem & $0.79(0.53-1.16)$ & 0.230
\end{tabular}

${ }^{1}$ BLI: b-lactamases inhibitors 


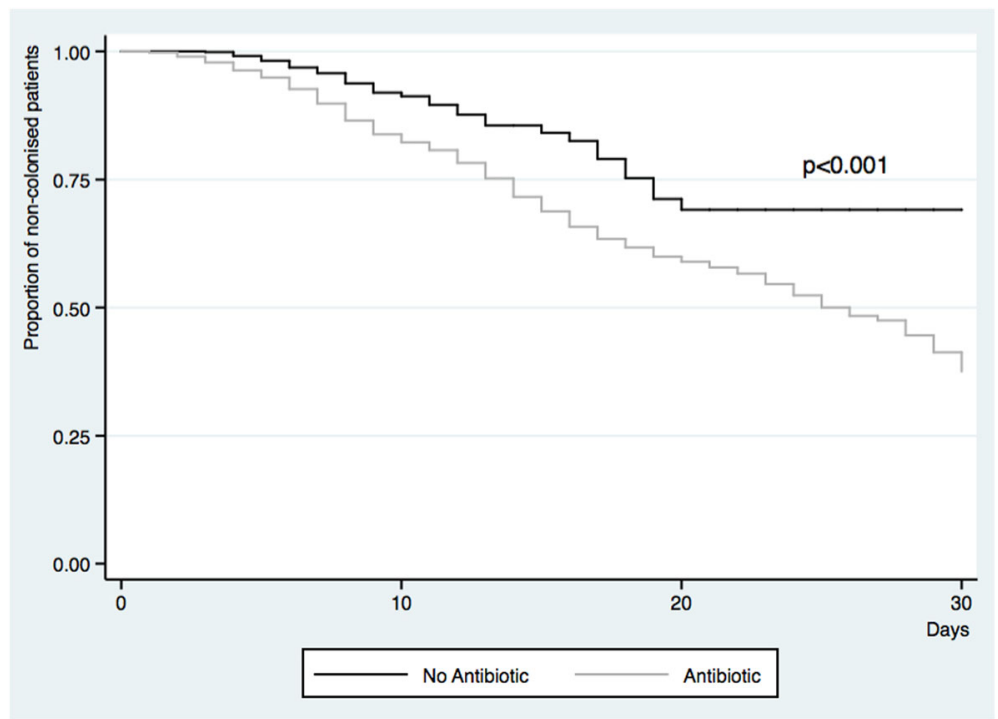

Fig. 2 Days until K. pneumoniae colonisation of the patients included based on the use of antimicrobials

rate of $K$. pneumoniae colonisation in these units has been little studied [20, 21]. Unlike previous studies [18-20], we included both rectal and respiratory samples which increased the probability of identifying patients colonised by these strains.

The results we obtained show that the length of stay in the ICU is a factor that determines colonisation by MR strains of K. pneumoniae, whose colonisation prevalence exceeded $50 \%$ in patients who remained in the unit for more than three weeks. However, we have also demonstrated that patients who received antibiotic treatments were colonised earlier, showing that, although the unit hospitalisation length inevitably conditions the risk of colonisation, the consumption of antibiotics is a key factor in accelerating it. Changes in the microbiota observed in critically ill patients $[22,23]$ could be favored by the use of antibiotics, which would bring on the selection and persistence of the most resistant strains. It should be noted that certain patients remain without being colonized despite having been admitted to the unit for several weeks and despite having received different antibiotic treatments.. It has been postulated that there could be factors specific to the flora of the host, which are currently not understood, that might act as protectors against exogenous strains [24].

Among the antimicrobials studied, use of the penicillin/BLI and aminoglycosides combination has been associated with a significant colonisation risk. The capacity of penicillins/BLIs to select MR strains is well known $[25,26]$. However, data referring to the use of aminoglycosides are more controversial and disparate results have so far been reported in the literature [17, 18, 25]. Carbapenems did not appear to have any significant effect on individual patient exposure or colonisation by MR strains. This phenomenon could be explained in part because in our unit, this antibiotic is usually used in patients who are already colonised by MR strains, and who completed their follow-up in our study.

Table 4 Relationship between antibiotic use (Daily Defined Dose) and colonization

\begin{tabular}{|c|c|c|c|c|}
\hline & \multicolumn{2}{|c|}{ \% Colonization during the same month } & \multicolumn{2}{|c|}{$\%$ Colonization during the next month } \\
\hline & Coefficient & $p$ value & Coefficient & $p$ value \\
\hline Carbapenem & 0.720 & 0.220 & 0.380 & $0.043^{*}$ \\
\hline Quinolones & -0.079 & 0.688 & -0.086 & 0.669 \\
\hline${ }^{1} \mathrm{BLI}$ & -0.048 & 0.856 & 0.065 & 0.787 \\
\hline Cephalosporin & -0.665 & 0.164 & 0.616 & $0.013^{*}$ \\
\hline AminoglycosidesAmynoglycosides & 0.392 & 0.472 & 0.833 & 0.226 \\
\hline Linezolid & 0.569 & 0.075 & 0.287 & 0.434 \\
\hline Total & -0.055 & 0.163 & 0.024 & 0.557 \\
\hline
\end{tabular}

${ }^{1} \mathrm{IBL}{ }^{1} \mathrm{BLI}$ : b-lactamases Inhibitors

*Statistically significant 
With respect to the pressure on antimicrobial consumption, very few studies have evaluated the overall effect of the use of antibiotics and colonisation by MR strains in ICU units [18]. In the present study, we found an association between the global consumption of carbapenems and cephalosporins, and colonisation in the month following the intervention. The effect produced by this group of antibiotics and their capacity to select for MR bacteria is known, having been related to a selective pressure on the microbiota [10, 26]. However, it should be noted that no association was found between the overall consumption of quinolones or BLIs and the number of colonised patients, even though these are also frequently cited in the literature as factors related to the selection of MR strains [9, 18, 27], and despite the fact we found an individual effect in the case of BLI. This current analysis shows that, although the consumption of antibiotics seems to lead to higher overall colonisation, analysis of the association between the antimicrobial-use pressure and the development of resistance is complex. It should be pointed out that the evaluation of the impact of antibiotics on in critically ill patients microbiota is highly complex because of the heterogeneity of dosing regimens, the co-administration of other antibiotics and other drugs that may also impact on its diversity. Therefore, to reduce the colonisation pressure, in addition to minimising exposure to antibiotics, it is essential to apply measures that have been shown to reduce colonisation from other patients or from colonised environmental surfaces; these measures include handwashing surveillance programs, adequate cleaning of patients, and the use of diverse strategies to minimise surface contamination $[28,29]$.

Among the limitations of the study is, first of all, its unicentric nature. The analysed data come from a medical unit with specific epidemiological characteristics, which may not be applicable to other units with other types of MR strains. This study has been focused on MR K. pneumoniae. Most of patients that have other MR bacteria, such MRSA, E. cloacae, P. aeruginosa o A. baumannii had been admitted to other units prior to their entry into the ICU, with a high suspicion that they could have been colonized in them. However, K. pneumoniae is an endemic bacteria of the ICU within our hospital, so we could study the effect of antibiotic use on the incidence of $K$ pneumoniae colonisation better than with the set of MR strains, with a lesser influence of external factors to those of the ICU.

We have presented an ICU we a high proportion of carbapenem-produced $K$. pneumoniae colonisation with a relatively low consumption of carbapenems, which reflects the endemic situation of the unit. It should be noted that, in an endemic situation, most patients are colonised through cross-transmission via several different materials and from the health professionals themselves [30]. This is why most patients may be initially colonised on a surface of their body before later presenting colonisation of their digestive tract, from where the surveillance samples are obtained. The effect of antibiotic consumption on this type of transmission is uncertain. However, the loss of the bacterial flora because of the use of antimicrobials could facilitate this colonisation phenomenon.

Although surgical intervention has been associated with higher colonisation by MR bacteria [31], we did not include this variable as a colonisation risk factor in our study because our ICU does not see surgical patients. Similarly, we did not study the impact of the insertion of central venous catheters on colonisation, even though other authors have demonstrated that this is a risk factor for infection by MR strains [12, 25], because most of the patients admitted to our unit have this type of line. On the other hand, some patients were evaluated during their stay in our unit but not subsequently followed-up in the hospitalisation rooms. This means that the true number of colonised patients may have been underestimated. In addition, during this period, even though our ICU had agreed-upon protocols that were well-known by the unit's staff, we were unable to rigorously analyse compliance with the various strategies in place to help prevent patient colonisation. This fact, together with the issue that the different measures were carried out at different times during the follow-up period, has prevented the effect of these interventions from being included in the analysis.

\section{Conclusion}

Given the association between colonisation and infection, and the increase in morbidity and mortality associated with infection with MR strains [6, 13], further strategies to minimise colonisation by these strains must be implemented. In this context, minimising patient exposure to antibiotics is a fundamental factor in reducing this type of colonisation. In summary, the individual and overall consumption of antibiotics determines the colonisation of patients by MR K. pneumoniae. Therefore, the implementation of strategies that reduce exposure are required to reduce colonisation by these types of MR strains.

\section{Abbreviations}

DDD: Daily defined dose; ECDC: European Centre for Disease Prevention and Control; ESBL: Extended-spectrum b-lactamases; ICU: Intensive care unit; MIC: Minimum inhibitory concentration; MR: Multiresistant; MRSA: Methicillinresistant S. aureus

\section{Acknowledgements}

None.

Funding

This research did not receive any specific grant from funding agencies in the public, commercial, or not-for-profit sectors.

Availability of data and materials

The datasets used and/or analyzed during the current study are available from the corresponding author on reasonable request. 


\section{Authors' contributions}

All authors have seen and approved the manuscript. Contributions were as follows: conception and design: JR, PR, AC; acquisition of data: MG, MAS, JF, EV AK; interpretation and analysis: MG, PR, JR; statistics: EV, JR. writing: PR, JR.

\section{Ethics approval and consent to participate}

The study had the approval of the Biomedical Research Ethics Committee of the Hospital La Fe (N IPA-ANT-2014-01).

\section{Consent for publication}

All the authors carefully read the manuscript and fully approve of it.

\section{Competing interests}

The authors declare that they have no competing interests.

\section{Publisher's Note}

Springer Nature remains neutral with regard to jurisdictional claims in published maps and institutional affiliations.

\section{Author details}

${ }^{1}$ Intensive Care Unit, IIS La FE, Hospital Universitario y Politécnico La Fe, Valencia, Spain. ${ }^{2}$ Microbiology Department, Hospital Universitario y Politécnico La Fe, Valencia, Spain. ${ }^{3}$ Intensive Care Unit, Hospital Universitario y Politécnico La Fe, Valencia, Spain.

\section{Received: 14 December 2018 Accepted: 31 January 2019}

\section{Published online: 14 February 2019}

\section{References}

1. Munoz-Price LS, Poirel L, Bonomo RA, Schwaber MJ, Daikos GL, Cormican M, et al. Clinical epidemiology of the global expansion of Klebsiella pneumoniae carbapenemases. Lancet Infect Dis. 2013;13:785-96.

2. Rojas $\amalg$, Weinstock GM, De La Cadena E, Diaz L, Rios R, Hanson BM, et al. An analysis of the epidemic of Klebsiella pneumoniae CarbapenemaseProducing K. Pneumoniae: convergence of two evolutionary mechanisms creates the «perfect storm». J Infect Dis. 2017;217:82-92.

3. Cartelle M, del Mar TM, Pertega S, Beceiro A, Dominguez MA, Velasco D, et al. Risk factors for colonization and infection in a hospital outbreak caused by a strain of Klebsiella pneumoniae with reduced susceptibility to expanded-Spectrum Cephalosporins. J Clin Microbiol. 2004;42:4242-9.

4. Coque TM, Baquero F, Canton R. Increasing prevalence of ESBL-producing Enterobacteriaceae in Europe. Euro Surveill. 2008;13.

5. Paterson DL, Ko W-C, Von Gottberg A, Mohapatra S, Casellas JM, Goossens $\mathrm{H}$, et al. International prospective study of Klebsiella pneumoniae bacteremia: implications of extended-spectrum beta-lactamase production in nosocomial Infections. Ann Intern Med. 2004;140:26-32.

6. Cosgrove SE. The relationship between antimicrobial resistance and patient outcomes: mortality, length of hospital stay, and health care costs. Clin Infect Dis. 2006;42(Suppl 2):S82-9.

7. Thaden JT, Li Y, Ruffin F, Maskarinec SA, Hill-Rorie JM, Wanda LC, Reed SD, et al. Increased costs associated with bloodstream infections caused by multidrug-resistant gram-negative Bacteria are due primarily to patients with hospital-acquired infections. Antimicrob Agents Chemother. 2017;61: e01709-16.

8. Demirdag K, Hosoglu S. Epidemiology and risk factors for ESBLproducing Klebsiella pneumoniae: a case control study. J Infect Dev Ctries. 2010;4:717-22.

9. Blanco N, Harris AD, Rock C, Johnson JK, Pineles L, Bonomo RA, et al. Risk Factors and Outcomes Associated with Multidrug-Resistant Acinetobacter baumannii upon Intensive Care Unit Admission. Antimicrob Agents Chemother. 2017:62

10. Huang Y, Zhuang S, Du M. Risk factors of nosocomial infection with extended-spectrum beta-lactamase-producing bacteria in a neonatal intensive care unit in China. Infection. 2007:35:339-45.

11. Sedláková MH, Urbánek K, Vojtová V, Suchánková H, Imwensi P, Kolář M. Antibiotic consumption and its influence on the resistance in Enterobacteriaceae. BMC Res Notes. 2014;7:454.

12. Vasudevan A, Mukhopadhyay A, Goh EY-Y, Li J, Tambyah PA. Risk factors for infection/colonization caused by resistant gram negative bacilli in critically ill patients (an observational study of 1633 critically ill patients). Prev Med. 2013;57(Suppl):S70-3.
13. Giannella M, Trecarichi EM, De Rosa FG, Del Bono V, Bassetti M, Lewis RE, et al. Risk factors for carbapenem-resistant Klebsiella pneumoniae bloodstream infection among rectal carriers: a prospective observational multicentre study. Clin Microbiol Infect. 2014;20:1357-62.

14. Magiorakos A-P, Srinivasan A, Carey RB, Carmeli Y, Falagas ME, Giske CG, et al. Multidrug-resistant, extensively drug-resistant and pandrug-resistant bacteria: an international expert proposal for interim standard definitions for acquired resistance. Clin Microbiol Infect. 2012;18:268-81.

15. European Committee on Antimicrobial Susceptibility Testing. 2013. EUCAST guidelines for detection of resistance mechanisms and specific resistances of clinical and/or epidemiological importance. Version 1.0, December 2013. European Committee on Antimicrobial Susceptibility Testing, Basel. http:// www.eucast.org/fileadmin/src/media/PDFs/EUCAST_files/Resistance mechanisms/EUCAST_detection_of_resistance_mechanisms_v1.0_20131211. pdf. Date of access: 02 Nov 2016.

16. WHO Global Strategy for Containment of Antimicrobial Resistance. Disponible en:http://www.who.int/drugresistance/WHO_Global_Strategy English.pdf. Date of access: 20 Nov 2016.

17. Trinh TD, Zasowski EJ, Claeys KC, Lagnf AM, Kidambi S, Davis SL, et al. Multidrug-resistant Pseudomonas aeruginosa lower respiratory tract infections in the intensive care unit: prevalence and risk factors. Diagn Microbiol Infect Dis. 2017:89:61-6.

18. Gómez-Zorrilla S, Camoez M, Tubau F, Periche E, Cañizares R, Dominguez $M A$, et al. Antibiotic pressure is a major risk factor for rectal colonization by multidrug-resistant Pseudomonas aeruginosa in critically ill patients. Antimicrob Agents Chemother. 2014:58:5863-70.

19. Munier A-L, Biard L, Rousseau C, Legrand M, Lafaurie M, Lomont A, et al. Incidence, risk factors, and outcome of multidrug-resistant Acinetobacter baumannii acquisition during an outbreak in a burns unit. J Hosp Infect. 2017:97:226-33

20. Pilmis B, Cattoir V, Lecointe D, Limelette A, Grall I, Mizrahi A, et al. Carriage of ESBL-producing Enterobacteriaceae in French hospitals: the PORTABLSE study. J Hosp Infect. 2018:98:247-52.

21. Akturk H, Sutcu M, Somer A, Aydın D, Cihan R, Ozdemir A, et al. Carbapenem-resistant Klebsiella pneumoniae colonization in pediatric and neonatal intensive care units: risk factors for progression to infection. Braz J Infect Dis. 2016:20:134-40.

22. Akrami K, Sweeney DA. The microbiome of the critically ill patient. Curr Opin Crit Care. 2018:24:49-54.

23. Latorre M, Krishnareddy S, Freedberg DE. Microbiome as mediator: do systemic infections start in the gut? World J Gastroenterol. 2015;21:10487-92.

24. Vollaard EJ, Clasener HA. Colonization resistance. Antimicrob Agents Chemother. 1994;38:409-14.

25. Cobos-Trigueros N, Solé M, Castro P, Torres JL, Hernández C, Rinaudo M et al. Acquisition of Pseudomonas aeruginosa and its resistance phenotypes in critically ill medical patients: role of colonization pressure and antibiotic exposure. Crit Care Lond Engl. 2015;19:218.

26. Zerr DM, Miles-Jay A, Kronman MP, Zhou C, Adler AL, Haaland W, et al. Previous antibiotic exposure increases risk of infection with extended-Spectrum- $\beta$ lactamase- and AmpC-producing Escherichia coli and Klebsiella pneumoniae in pediatric patients. Antimicrob Agents Chemother. 2016;60:4237-43.

27. Defez C, Fabbro-Peray P, Bouziges N, Gouby A, Mahamat A, Daurès JP, et al. Risk factors for multidrug-resistant Pseudomonas aeruginosa nosocomial infection. J Hosp Infect. 2004:57:209-16.

28. Zaky A, Zeliadt SB, Treggiari MM. Patient-level interventions to prevent the acquisition of resistant gram-negative bacteria in critically ill patients: a systematic review. Anaesth Intensive Care. 2015:43:23-33.

29. Musuuza JS, Sethi AK, Roberts TJ, Safdar N. Implementation of daily chlorhexidine bathing to reduce colonization by multidrug-resistant organisms in a critical care unit. Am J Infect Control. 2017;45:1014-7.

30. Gurieva T, Dautzenberg MJD, Gniadkowski M, Derde LPG, Bonten MJM, Bootsma MCJ. The transmissibility of antibiotic-resistant Enterobacteriaceae in intensive care units. Clin Infect Dis. 2018;66:489-93.

31. Vasudevan A, Mukhopadhyay A, Li J, Yuen EGY, Tambyah PA. A prediction tool for nosocomial multi-drug resistant gram-negative bacilli infections in critically ill patients - prospective observational study. BMC Infect Dis. 2014; $14: 615$ 